\title{
REVUE
}

\section{L'INDUSTRIE LAITIÈRE EN ANGLETERRE ET LA POLITIQUE DU GOUVERNEMENT BRITANNIQUE}

par

\author{
G. GÉEIN
}

Ingénieur I. E. P. C.

Il résulte d'informations qui viennent d'être publiées que le Gouvernement britannique ne va pas tarder à définir maintenant quelle va être sa politique en ce qui concerne le soutien à apporter à l'industrie laitière, politique à longue échéance qui tiendra certainement compte des conclusions du rapport qui a été publié il y a quelques mois par la Commission de réorganisation de l'industrie laitière en Grande-Bretagne.

Les recommandations qui figurent dans ce rapport intéressent toutes les branches de l'industrie des produits alimentaires et aujourd'hui, alors que le travail de cette Commission a été largement discuté par tous les cercles intéressés, il peut être utile d'indiquer quelles furent les recommandations de cette Commission.

Rappelons tout d'abord que cette Commission, composée de 5 membres, a été nommée par le Gouvernement dans le but de procéder à un contrôle des opérations, des différents organisateurs et contrôleurs du marché du lait en Grande-Bretagne et s'il y a lieu d'émettre des recommandations en vue d'améliorer le fonetionnement de ces organismes et d'assurer une meilleure coordination entre eux. Evidemment, un tel programme pouvait être diversement interprété et on pouvait en particulier concevoir qu'une étude de ce genre devait englober de très vastes questions.

Le rapport établi par la Commission indique que celle-ci a procédé à une très vaste enquête et s'est efforcée en particulier de recueillir l'opinion des représentants de toutes les organisations intéressées par les questions laitières, ainsi que celles d'un grand nombre d'individus, depuis les fermiers qui produisent le lait jusqu'aux consommateurs, même les plus modestes. La majeure partie du rapport consiste done en une étude critique des informations ainsi recueillies. On discute les diverses méthodes qui sont à la base de l'organisation du marché du lait actuel et on montre comment le marché pourrait être amélioré au bénéfice des consommateurs. Dans une dernière partie, les membres de la Commission ont brièvement résumé leur's recommandations.

La publication de ce rapport a fait beancoup de bruit et cela n'est pas étonnant, étant donné l'importance des intérêts auxquels 
il touche. Aussi, dès la publication, il y a quelques mois, de ce travail, des réactions très vives ont eu lieu, en particulier à la suite des propositions un peu exceptionnelles faites par la Commission.

Rappelons que parmi ces propositions, la principale a pour objet la création d'une Commission permanente du lait qui constituerait une autorité centrale pour l'industrie laitière, dont les décisions seraient impartiales et qui auraient en particulier à eharge d'administrer les fonds votés par les Chambres pour venir en aide à l'industrie, de fixer les prix et coordonner les différents intérêts des producteurs, transformateurs et consommateurs. La fixation des prix devrait être faite dans des conditions telles que le marché pourrait disposer régulièrement des quantités de lait qui lui sont nécessaires, mais le prix de vente ne serait pas déterminé comme actuellement par des marchandages entre producteurs et consommateurs, mais d'après des données résultant de l'expérience passée et de certaines estimations concernant la production actuelle.

On sait qu'actuellement le lait est vendu à certains gros transformateurs et producteurs de dérivés laitiers à des cours qui correspondent à une perte pour le producteur; cette perte est actuellement compensée par une augmentation excessive du prix de vente du lait aux petits consommateurs. Dans l'opinion de la Commission, cette perte devrait être supportée par l'Etat. D'autres recommandations de la Commission concernent également l'administration des différents organismes chargés de l'organisation du marché.

Dès la publication de ce rapport en novembre, dernier, les organisations d'agriculteurs furent unanimes à prendre position contre. Cette politique peut sembler étonnante, mais il ne faut pas oublier que l'organisation, la création des offices chargés de contrôler le marché du lait étaient dues aux Associations agricoles. C'est à ces Associations que l'on devait la création de la législation existante qui avait permis en particulier aux petits fermiers de surmonter les difficultés résultant de la crise agricole et qui avait renforcé leur situation devant les gros industriels. Ayant ainsi établi des organisations qui avaient fonctionné d'une façon à peu près satisfaisante, en dépit de nombreuses difficultés, il n'est pas étonnant que tous ces fermiers ne voyaient pas d'un oil favorable la création d'un Conseil supérieur, permanent et entièrement à la solde du Gouvernement.

Par contre, on a pu s'étonner de cette opposition de la part des producteurs à un programme qui prévoyait une aide gouvernementale, aide dont certains avaient fixé le montant à environ 5 millions de livres. A cela, les producteurs répliquent que l'assistance 
de l'État, telle qu'elle est proposée, n'améliorerait pas leur situation, et que c'est surtout le petit consommateur qui en bénéficierait en payant son lait moins cher. Dans l'esprit des membres de la Commission, une diminution du prix de vente du lait devrait avoir pour objet d'augmenter la consommation de ce produit.

En résumé, on se trouve encore actuellement en face de l'opposition de producteurs qui craignent de perdre le contrôle de leur propre industrie. Ces producteurs ne veulent pas entendre parler de contrôle de la production, de fixation de prix par une Commission gouvernementale et cette politique n'est pas étonnante, car on sait que de nombreux autres industriels appartenant à des branches les plus diverses ont toujours été opposés à un contrôle quel qu'il soit lorsqu'il est réalisé par des personnes prises en dehors de leur sein.

Le problème se complique encore du fait que la quantité totale de lait consommé en Angleterre représente beancoup plus que la quantité produite dans le pays. L'Angleterre a toujours été un débouché intéressant pour les produits agricoles, en particulier pour le lait et les produits laitiers, produits en excès dans les autres pays européens.

Quoi qu'il en soit, le Gouvernement est done à la veille de prendre des décisions définitives et le contrôle qu'il a l'intention d'exercer devra également porter sur le lait et sur les produits laitiers importés de l'étranger et des Dominions, d'où la fixation des droits de douane, etc.

A ces conditions d'ordre économique, il faudra également ajouter d'autres conditions, afin que l'intérêt des consommateurs soit totalement sauvegardé en ce qui concerne la santé publique. Le contrôle devra porter sur la qualité du lait au point de vue bactériologique, afin d'éviter la propagation de la tuberculose et d'autres maladies et cela entraînera probablement une vérification beaucoup plus stricte et sévère qu'actuellement des installations de pasteurisation.

Le Ministère de l'Agriculture n'a pas une tâche aisée à remplir quand on songe aux intérêts si opposés des producteurs de lait, des distributeurs, des consommateurs, des fabricants de produits laitiers et enfin des fabricants et importateurs de produits étrangers. Il désire par les décisions prises défendre l'agriculture britannique et favoriser l'achat de produits d'origine anglaise. 